\title{
DA No Longer Lost in Translation: Evidence that Google Translate Works for Comparative Bag-of-Words Text Applications
}

\section{Erik de Vries', Martijn Schoonvelde ${ }^{2}$ and Gijs Schumacher ${ }^{3}$}

\author{
${ }^{1}$ Department of Media and Social Sciences, University of Stavanger, Stavanger, Norway.Email: erik.devries@uis.no \\ ${ }^{2}$ Department of Political Science and Public Administration, Vrije Universiteit, Amsterdam, The Netherlands. \\ Email:h.j.m.schoonvelde@vu.nl \\ ${ }^{3}$ Department of Political Science, University of Amsterdam, Amsterdam, The Netherlands. Email: g.schumacher@uva.nl
}

\begin{abstract}
Automated text analysis allows researchers to analyze large quantities of text. Yet, comparative researchers are presented with a big challenge: across countries people speak different languages. To address this issue, some analysts have suggested using Google Translate to convert all texts into English before starting the analysis (Lucas et al. 2015). But in doing so, do we get lost in translation? This paper evaluates the usefulness of machine translation for bag-of-words models-such as topic models. We use the europarl dataset and compare term-document matrices (TDMs) as well as topic model results from gold standard translated text and machine-translated text. We evaluate results at both the document and the corpus level. We first find TDMs for both text corpora to be highly similar, with minor differences across languages. What is more, we find considerable overlap in the set of features generated from human-translated and machine-translated texts. With regard to LDA topic models, we find topical prevalence and topical content to be highly similar with again only small differences across languages. We conclude that Google Translate is a useful tool for comparative researchers when using bag-of-words text models.
\end{abstract}

Keywords: automated content analysis, statistical analysis of texts, bag-of-words models, Google Translate, LDA

\section{Introduction}

Automated text analysis is like a gold rush. Many researchers have noticed its potential and are now using methods such as topic modeling, scaling and sentiment analysis to analyze political texts (for an overview see Grimmer and Stewart 2013). But researchers interested in cross-country comparisons face a problem: people speak different languages. In order to make comparisons across countries, researchers first need to translate texts from several languages into one. On the plus side, nowadays this can be automated by using machine translation, such as Google Translate. But does the meaning of these texts get lost in Google translation? That is, do we lose (too much) information if we Google Translate texts before we analyze them? Or does doing so leave us like the poor souls who journeyed west for gold but were left with nothing?

This paper evaluates the usefulness of machine translation for automated bag-of-words models. ${ }^{1}$ We identify and evaluate four reasons why the meaning of a text may get lost

Edited by

Jonathan N. Katz

(c) The Author(s) 2018. Published by Cambridge University Press on behalf of the Society for Political Methodology. This is an Open Access article, distributed under the terms of the Creative Commons Attribution licence (http://creativecommons.org/ licenses/by/4.0/), which permits unrestricted re-use, distribution, and reproduction in any medium, provided the original work is properly cited.
Authors' note: Replication code and data are available at the Political Analysis Dataverse (De Vries, Schoonvelde, and Schumacher 2018) while the supplementary materials for this article are available on the Political Analysis web site. The authors would like to thank James Cross, Aki Matsuo, Christian Rauh, Damian Trilling, Mariken van der Velden and Barbara Vis for helpful comments and suggestions. GS and MS acknowledge funding from the European Union's Horizon 2020 research and innovation program under grant agreement No 649281, EUENGAGE. EdV acknowledges funding for a research assistantship from the Access Europe (since 2018: UVAccess Europe) research center at the University of Amsterdam.

1 The goal in this paper differs from much work in computational linguistics or Natural Language Processing (NLP), as that type of research is mostly concerned with syntax, readability and the correct use of grammar in translations (e.g., Scarton and Specia (2014), Kaljahi and Samad (2015), Aharoni (2015)). In contrast, this paper compares bag-of-words vectors and topic models that are based on them. Both are used regularly in applications of automated text analysis in the social sciences. 
in translation. First, a general problem occurs when words or stems in machine-translated documents are translated differently than those in gold standard documents, leading to different term-document matrices (TDMs). ${ }^{2}$ We evaluate this issue by comparing the overlap between gold standard and machine-translated TDMs. Other translation problems relate more specifically to LDA topic modeling, a popular bag-of-words model that identifies the topics in a corpus, and assigns documents and words to these topics. In this case, translation issues may arise because (1) topics might be distributed differently in the machinetranslated corpus than in the gold standard corpus, (2) machine-translated documents are assigned to different topics than gold standard documents and (3) a topic in the machine-translated corpus consists of different words than the same topic in the gold standard corpus. We evaluate each issue by systematically comparing topic models estimated using machine-translated documents with those estimated using human-translated (gold standard) documents.

To set up our comparisons, we use the europarl dataset (Koehn 2005), which contains the official transcriptions of debates in the European Parliament both in English and in most other official languages of the EU. From this dataset we take debate transcriptions in Danish, German, Spanish, French and Polish for the period of January 2007 to November 2011. Delivered by professional translators, these official transcriptions serve as our gold standard. ${ }^{3}$ We first compare the bag-of-words vectors of each document in the machine translation and the gold standard translation. We then compare the output of the LDA topic models in three ways: topical prevalence at the document level, topical prevalence at the corpus level and topical content at the corpus level. ${ }^{4}$

We find that TDMs for both sets of data are highly similar, with significant but minor differences across languages. What is more, we find considerable overlap in the set of features (stems) appearing in human- and machine-translated texts. With regards to LDA topic models, at both the document and the corpus levels we find topical prevalence to be similar with only small differences across languages, and we find topical content to strongly overlap as well. These findings suggest that Google Translate does in fact generate useful TDMs, and, what is more, it deals successfully with the above-mentioned risks of machine translation when estimating topic models. We conclude that Google Translate is a useful tool for researchers who use or want to use bag-of-words text models for comparative questions.

\section{Background}

Numerous bag-of-words based studies have analyzed machine-translated texts, yet little is known about the quality of machine translations and its impact on subsequent analyses. Generally, authors either assume machine-translated text to be suitable for their purposes or they do not pay attention to the issue at all. For example, Agarwal et al. (2011) use Twitter data which was machine-translated by an unidentified commercial source, but they do not address the possibility that machine translation may have influenced their results. Schwarz, Traber, and Benoit (2017) use Google Translate in the multilingual Swiss context. While these authors describe the machinetranslation process in more detail, they do not discuss comparisons between different machinetranslation strategies, or the quality of their translations.

To be clear, we do not imply that machine translation is not useful for analyzing texts in multiple languages. As Lotz and Van Rensburg (2014) show, developments in machine-translation systems

2 Throughout the paper, we use the terms bag-of-words vectors and term-document matrices (TDMs) interchangeably.

3 Partly because of thorough quality requirements, the costs of hiring professional translators in the European Union are high, by some estimates as much as $€ 2$ per EU inhabitant per year (see http://ec.europa.eu/dgs/ translation/faq/index_en.htm). A gold standard indeed.

4 Topical prevalence refers to which topics appear in a document or in the corpus as a whole (i.e., topic distributions), whereas topical content refers to what words constitute a topic (i.e., word distributions). (Lucas et al. 2015). 
are moving fast and their quality is clearly increasing with time. Balahur and Turchi (2014) give a comprehensive account of using machine-translated text for automated analyses in the context of sentiment analysis, and Courtney et al. (2017) find that machine-translated newspaper articles can be reliably categorized by human coders. But while these contributions are highly relevant, they do not evaluate the implications of machine translation for bag-of-words methods more generally. The same is true for Lucas et al. (2015), who write extensively about the possible pitfalls of analyzing machine-translated text but do not evaluate its quality empirically. Adding to this line of research, this paper systematically evaluates both the bag-of-words approach in general and topic modeling in particular.

Another issue relevant to this study concerns the impact of specific languages and language groups on machine-translation quality. For example, machine-translated texts may be of better quality when translated from French to English than when translated from Polish to English. There are two reasons for this. First, some language pairs are simply more easily translated than others (Koehn and Monz 2006). Furthermore, larger parallel corpora are available to train machine-translation models for some language pairs than for others (e.g., there is more parallel data available for French and English than there is for Polish and English). To examine this possibility we include in our analysis languages from different language groups: French and Spanish (belonging to the Italic language group), German and Danish (belonging to the Germanic language group), and Polish (belonging to the Balto-Slavic language group). ${ }^{5}$

\section{Data and Measurement}

To evaluate the quality of machine translation, we need to compare its output to gold standard translations of identical documents. ${ }^{6}$ The europarl dataset (Koehn 2005) contains parallel corpora which allow us to set up such comparisons. The dataset consists of official European Parliament debate transcriptions in most of the official EU languages. ${ }^{7}$ The europarl dataset ranges from April 1996 until November 2011, but some Slavic language translations were included only since January 2007. Because of that, we focus our analysis on translations between 2007 and 2011.

The primary purpose of the europarl dataset is to train, test and improve machine-translation algorithms (e.g. Koehn 2005; Popescu-Belis et al. 2012; Loaiciga, Meyer, and Popescu-Belis 2014). The data is available in both the raw form and as text files with sentence-aligned language pairs. We use the raw data, because the sentence-aligned text files do not distinguish between different dates and debate chapters. The raw data files are organized per session (typically one day) and chapter. Each chapter is a different item on the agenda (e.g a debate, questioning of EU official or vote) of a session. ${ }^{8}$ When estimating topic models, we consider each chapter to be a single document, because each chapter in a session concerns a specific agenda item. Each agenda item may in turn consist of multiple topics.

5 While there are different topologies of language groups in the field of linguistics, this paper uses the topology described in Gray and Atkinson (2003).

6 Replication code and data are available at the Political Analysis Dataverse (De Vries, Schoonvelde, and Schumacher 2018) while the Supplementary materials for this article are available on the Political Analysis web site.

7 Contributions to debates in the European Parliament can be either in English or in one of the other official EU member state languages. Contributions in those languages are then translated-by official translators-in all other recognized EU languages either directly or indirectly through English. What we consider our gold standard data of the debates is the English corpus which consists of (1) English contributions, (2) contributions in one of the EU languages translated into English by official translators. What we consider our machine-translated data consists of Google translations into English of these same contributions in (1) one of our five languages and (2) in other EU languages that have been translated into these five languages either directly or indirectly by official translators.

8 Because the provided data is not exactly the same for all languages (e.g. Chapter 5 in the session of 04-01-2007 might be present in the English but not in the German data, while the German data does contain other chapters from that same session), we had to match all language pairs (EN-DA, EN-DE, EN-ES, EN-FR, EN-PL) by checking for the presence of each chapter in each session for both languages. This results in between 2148 (DE) and 2347 (FR) chapters per language pair. 


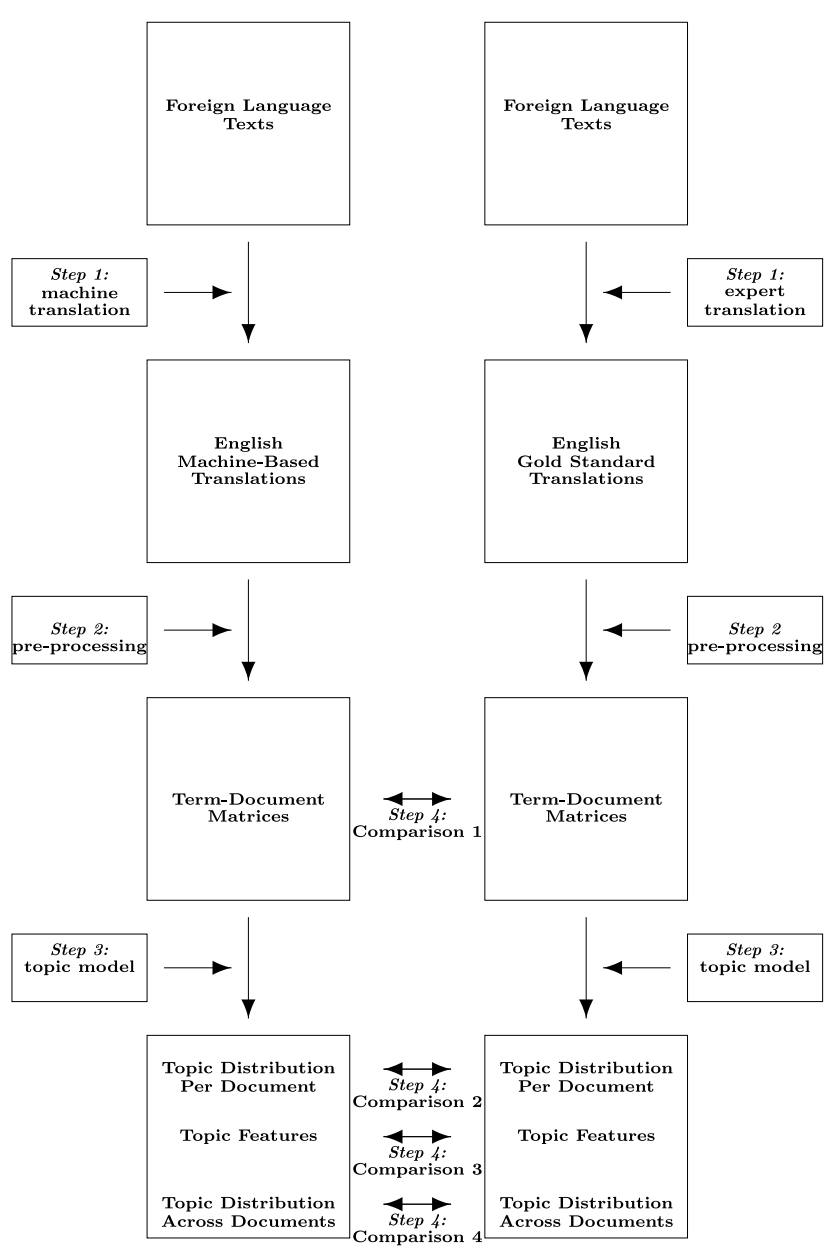

Figure 1. Research design.

Note: This figure shows the different steps of our research design. In both cases we start with non-English texts, which have been translated into English, either through Google Scholar or through EU-employed expert translators (Step 1). The English translations are then preprocessed and turned into TDMs (Step 2), on which we then estimate a topic model (Step 3). We then compare our four different outcome variables (Step 4). The comparisons are the following: Comparison 1: document-to-document comparison TDM similarity; Comparison 2: document-to-document comparison of topic distributions (topical prevalence); Comparison 3: topic-to-topic comparison of stem weights (topical content); Comparison 4: topic-to-topic comparison of topic distribution (topical prevalence).

\section{Methods}

Figure 1 shows the steps we take to compare machine-translated and gold standard documents. In both cases we start with identical non-English texts, which have been translated into English, either through Google Translate or through EU-employed expert translators (Step 1). These translations are preprocessed and turned into TDMs (Step 2) on which we then estimate a topic model (Step 3). We then compare the similarities of the TDMs, the topical prevalence at the level of individual documents and the corpus at large, and the topical content (Step 4). In what follows we discuss each step in more detail.

\section{Step 1. Machine translation and Google Translate}

We use Google Translate as the specific machine-translation service to evaluate the performance of machine-translated texts in bag-of-words analyses. We chose Google Translate because of its translation quality, which is top-tier when compared to other online machine translating services (Hampshire and Salvia 2010). We translated the texts using the Google Website Translator plugin 
which can translate web pages. To be able to use this plugin we converted the raw text data to bare html web pages. The translation process took place in August and September 2016. ${ }^{9}$ We have translated the texts into English, because machine-translation algorithms are expected to perform best when translating to and from English. ${ }^{10}$

\section{Step 2. Preprocessing and generating TDMs}

When using bag-of-words models, it is common to preprocess the data in order to remove noise. In our case we have removed punctuation, numbers and general stopwords, and all remaining words have been lowercased and stemmed. The preprocessing steps on both the gold standard and machine-translated texts are identical, and were applied to the translated texts. ${ }^{11}$ To perform these preprocessing steps, we used both Python and R libraries. For stemming, stopword removal, number removal, lowercasing, and punctuation removal, we used regular expressions in Python and the NLTK package (Bird, Klein, and Loper 2009). To create the TDMs we switched to R and the quanteda package (Benoit and Nulty 2013). ${ }^{12}$ We compare the TDMs of the machine-translated and gold standard documents and we also use them as input for the topics models described below. Readers primarily interested in our analysis of the TDMs may decide to skip the next section, which contains more technical details regarding the specification of our topic models.

\section{Step 3. Fitting topic models}

To assess the quality of machine-translated texts, we estimated topic models on the gold standard and machine-translated texts separately using the LDA algorithm (Blei, Ng, and Jordan 2003) and Gibbs sampling. For this we used the LDA function in the R topicmodels package (Hornik and Grün 2011). LDA is a generative model. It takes the words in each text as input and then estimates the topical prevalence and topical content in the corpus. To run the model researchers need to set a few parameters: the number of topics in the corpus, the model seed, burn-in time, the number of iterations and which and how many iterations to keep for use in the final model. To ensure that differences between a model based on the gold standard corpus and a model based on the machine-translated corpus are solely the result of language differences between these corpora, the parameters for the topic models based on gold standard translations and machine translations were kept identical. This means that the number of topics was kept constant, and a fixed seed was used-based on the sys.time variable-as suggested by Hornik and Grün (2011). This seed (1473943969) has been used for all models described below. Furthermore, the burn-in (1000) and number of iterations (300) were also kept constant. The algorithm keeps every 100th model and returns the model with the highest posterior likelihood (the best-fitting model). Consequently, all variation between the models-when the model parameters are kept the same-results from differences caused by the translation process.

The most important parameter to set is the number of topics in the topic model. This is crucial because the number of topics affects the distribution of words over topics (topical content) and the

9 We conducted the translations before Google rolled out their deep learning/neural network-based translation algorithms. Because this may have improved the quality of Google Translate, our results are likely to be conservative.

10 The reason for this is that English is the lingua franca of the internet and, by consequence, most translations are from or to English. This produces large parallel corpora between English and other languages. Machine-translation algorithms are trained on these models, and typically the more data the better the performance of the algorithm.

11 Recent research shows that seemingly innocuous preprocessing steps might impact the outcome of (unsupervised) automated text analyses (Denny and Spirling 2018; Greene et al. 2016). Our comparison, however, is between gold standard and machine-translated texts on which we applied identical preprocessing steps. Although we cannot be certain, we do not expect these preprocessing steps to have had a systematically different impact on both corpora. We should note, however, that, in general, the removal of stopwords will influence word and topic distributions within a topic model, and this will also apply to the model results we present here. But since stopwords contain-by definition-no topical content we expect their removal to have had minimal substantive implications.

12 We switched from Python to R for practical reasons only: we started this project using Python because of the large amount of scraping and cleaning required. We then switched to $\mathrm{R}$ because we considered it more suitable for the modeling and plotting tasks at hand. 


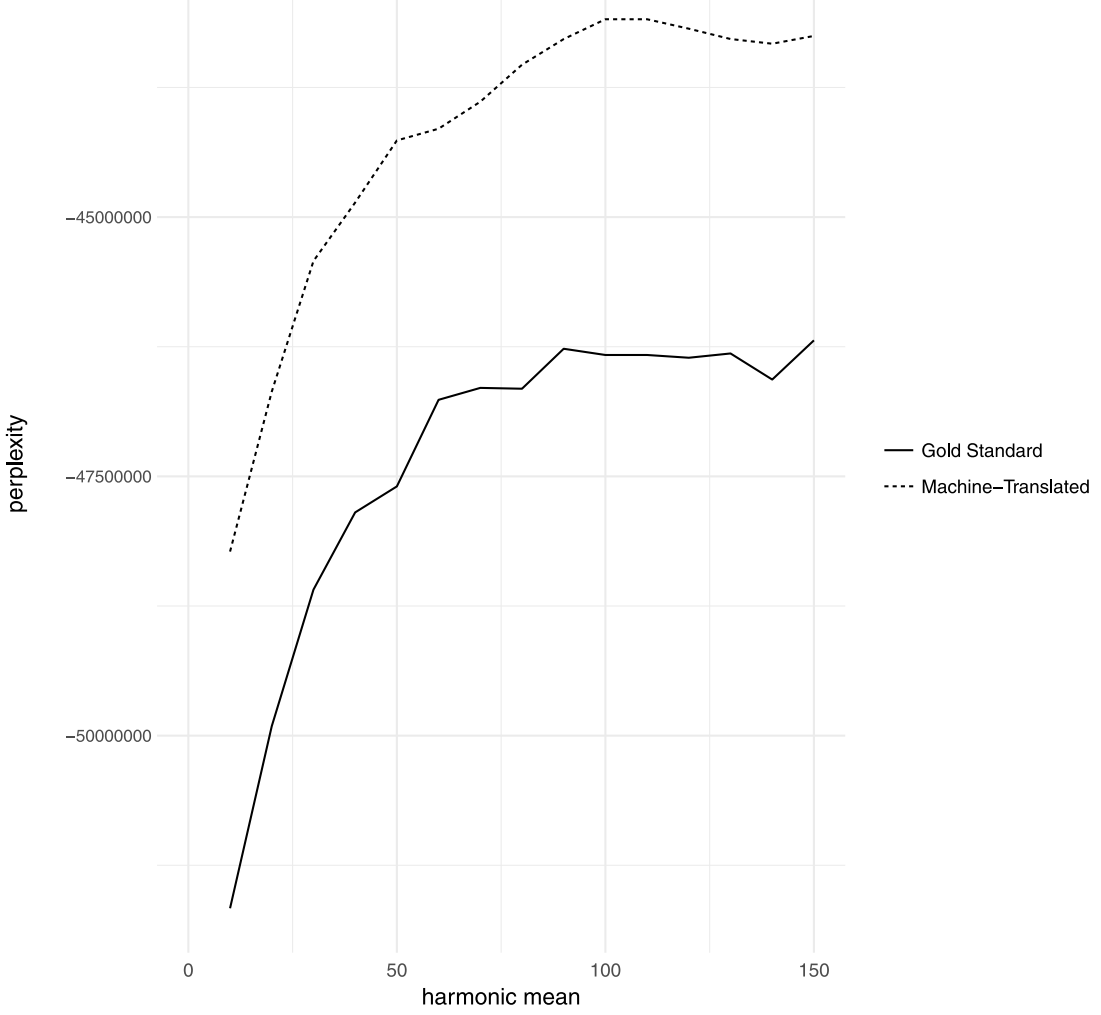

Figure 2. Model harmonic mean.

distribution of topics over documents (topical prevalence). When the number of topics changes so do these distributions. It was practically infeasible to run and optimize the number of topics for each language pair. Also, all language pairs are based on roughly similar data from the same time period. Therefore the optimum number of topics for all models was determined based on the French dataset. This is the largest gold standard and machine-translated dataset. We estimated the best-fitting number of topics by evaluating the model harmonic mean of models that contain between 10 and 150 topics, in increments of 10 . The model harmonic mean indicates the extent to which word counts in the documents used to construct the model match the word distributions in the model itself. Put differently, it indicates the extent to which the model accurately describes the distribution of words in the documents. In this case, a larger harmonic mean indicates that the model fits the data better. The results of the optimization runs are displayed in Figure 2. The gold standard model has an optimum of 90 topics. After 90 topics adding more topics does not improve model fit. The machine-translated model peaks at 100 topics. To isolate the effect of language differences between gold standard and machine-translated texts it is important to choose the same number of topics for both models. Therefore, we settled for 90 topics. That said, we also evaluated comparisons of models with 90 topics for the gold standard models and 100 topics for the machine-translated models. This produced results almost identical to the topic model comparisons with 90 topics. These results are available in the Supplementary Appendix.

Our next challenge is to match the topics generated by the gold standard and machinetranslated models. This is because the topic order in both models may differ (i.e., topic 1 in the machine-translated model may match best with, for example, topic 2 in the gold standard model). Our matching procedure is as follows: for each stem we find the highest loading in the machine-translated topic model and the gold standard topic model. For example, take the stem "agricultur". This stem loads highest on (is most important in) topic 12 of the machine-translated 
Table 1. Comparisons between gold standard and machine-translated data.

$\begin{array}{lll} & \text { Stems } & \text { Topics } \\ \text { Document level } & \text { Stem counts per document pair } & \text { Topic distribution per document pair } \\ \text { Corpus level } & \text { Stem loadings per topic pair } & \text { Topic distribution per topic pair }\end{array}$

model, and topic 45 in the gold standard model. This results in a 12-45 topic pairing for that specific stem. We subsequently count the topic pairings of all shared stems. We match topics based on the highest count of topic pairings. For example, we pair topic 12 of the machine-translated model with topic 45 in the gold standard model because they have the highest number of important, shared stems like the stem "agricultur" (see the Supplementary Appendix for a numerical example of our topic matching procedure). ${ }^{13}$ Using this procedure we matched 90 topics for the German corpus and 89 topics for all other languages. ${ }^{14,15}$

\section{Step 4. Comparing term-document matrices and topic models}

We make four different comparisons, which vary on two dimensions: stems versus topics and documents versus corpora (see Table 1). The comparison of TDMs takes place at the level of stems and documents (Comparison 1 in Figure 1). Furthermore, we report three comparisons based on our topic models, all of which give us evidence on how much the matched topics in the machine-translated and the gold standard topics overlap in content and prevalence. We evaluate topical content by means of stem loadings per topic pair (Comparison 3 in Figure 1). We evaluate topical prevalence by means of topic distributions over document pairs (Comparison 2 in Figure 1), and topic distributions across the corpus at large (Comparison 4 in Figure 1).

It is important to evaluate results at both the document and the corpus level because the former only speak to how similar individual documents are being characterized by the topic model (i.e., the extent to which topical prevalence for gold standard and machine-translated documents is similar). However, such a comparison does not tell us how similar the fitted topics themselves are. For example, both the gold standard and machine-translated document might have a high topic loading on topic 1, making them highly similar on the document level, but if topic 1 is about cars in the gold standard topic model and about trees in the machine-translated model, then document-level similarity does not tell us much. While the chances of this happening are slim, structural and consistent translation errors by Google Translate might cause such differences. As a consequence, the level of topical similarity does say something about the quality of the translation. We thus need comparisons on both the document and corpus level.

Our outcome measure for the TDM comparisons is different from that of the topic model comparisons. For the TDM comparisons, we use cosine similarity because-in contrast to correlation-it takes into account the absolute differences in values. This is relevant for comparing TDMs because of our goal of knowing how similar the counts of all TDM features per document pair are to each other. Cosine similarity varies between 0 and 1 , with the latter indicating a

13 It is of course possible that a topic in the machine-translated model is matched to several different topics in the gold standard model. For example, while "agricultur" is matched 12-45, it could be that the stem "farmer" loads highest on topics 12 and 33, resulting in two different topic pairings for topic 12 in the machine-translated model (namely 12-45 and 12-33). In those cases, we use the topic combination with the highest number of topic pairings, while ignoring the other. This results in topic pairs that always consist of the two topics that share their highest loading words with each other.

14 The reason that not all topics can be matched for all languages is because when every shared stem loads higher on another topic in the same model, there are simply no stems to base a match on. We can again take "agricultur" as an example. This stem is the most important (highest loading) in both topic 12 (word loading: 0.12) and 19 (word loading: 0.09). Yet our procedure only registers on which topic "agricultur" loads highest (which in this case is topic 12). So topic 19 will not be matched to another topic based on this stem alone. If not a single stem loads highest on a topic, then that topic cannot be matched and we discard it. In practice this means that while the unmatched topics might have some substantive importance, all their stems are-by design-more important to other topics.

15 Even though our matching procedure worked well, we should note there are other possible ways to match topics, using for example the Hungarian algorithm (see e.g., Chuang et al. 2013; Roberts, Stewart, and Airoldi 2016). 
Table 2. Cosine similarity distribution per language.

$\begin{array}{lccccc}\text { Language } & \text { N } & \text { Mean } & \text { St. Dev. } & \text { Min } & \text { Max } \\ \text { Danish } & 2,301 & 0.915 & 0.063 & 0.549 & 0.992 \\ \text { German } & 2,148 & 0.915 & 0.074 & 0.488 & 0.991 \\ \text { Spanish } & 2,335 & 0.929 & 0.059 & 0.483 & 0.991 \\ \text { French } & 2,347 & 0.925 & 0.064 & 0.564 & 0.989 \\ \text { Polish } & 2,338 & 0.913 & 0.073 & 0.475 & 0.989 \\ \text { Total: } & 11,469 & 0.919 & 0.066 & 0.475 & 0.992\end{array}$

Note: Statistically significant but substantively small difference between languages (ANOVA results: $\left.\mathrm{F}(4,11464)=27.855, \rho<0.001, \eta^{2}=0.010\right)$.

perfect match (i.e., two identical vectors). For the topic model comparisons, correlations are a more suitable similarity measure because they detect trends rather than absolute values. This is important because we make comparisons between different models. ${ }^{16}$ Correlations vary between -1 and 1 , with the latter indicating a perfect linear positive relationship, and the former indicating a perfect linear negative relationship.

\section{Results}

This section contains the results of our four comparisons, starting with the TDM analysis, and continuing with the topic model analyses.

\section{Comparing TDMs}

We first compare-at the document level-machine-translated and gold standard bags of words to each other, using the built-in similarity function in the quanteda R package (Benoit and Nulty 2013). Figure 3 displays the distribution of the cosine similarity scores for each language. Most notably, the average similarity between the gold standard documents and their machine-translated counterparts is very high $(M=0.92, S D=0.07)$. Furthermore, more than $92 \%$ of all document pairs achieve a cosine similarity score of 0.80 or higher. These results show that the TDMs of machine-translated and gold standard documents are very similar. Very often the stems in the machine-translated and gold standard documents occur with (approximately) the same frequency.

Table 2 shows the means and standard deviations for document cosine similarity scores per language. The differences between languages are tiny: the lowest mean cosine similarity (Polish $=0.913$ ) is only 0.016 smaller than the highest mean cosine similarity (Spanish $=0.929$ ). The French and Spanish documents have significantly higher average cosine similarities than the overall mean (French: $t=7.07, p<0.001$; Spanish: $t=5.11, p<0.001$ ), but the size of these differences is, again, very small (French: 0.005 and Spanish: 0.009). The Danish, Polish and German cosine similarities between document pairs are not significantly different from the overall mean.

We also consider the total number of unique stems (features), as well as the number of shared stems between the gold standard and machine-translated TDMs. The higher the number of shared stems, the more overlap there is. Figure 4 shows that the shared features of the TDMs of the gold standard and machine-translated documents overlap to a large degree (about $75 \%$ or higher). The number of shared features is also quite similar for each language (DA, 28431; DE, 27732; ES, 28578; FR, 28162; PL, 26916). The same goes for the features that are unique to either the gold standard or machine-translated TDMs.

16 As discussed before, changing the number of topics influences both the document-level and corpus-level topic distributions as well as stem distributions per topic, and because of that absolute values are no longer meaningful. 

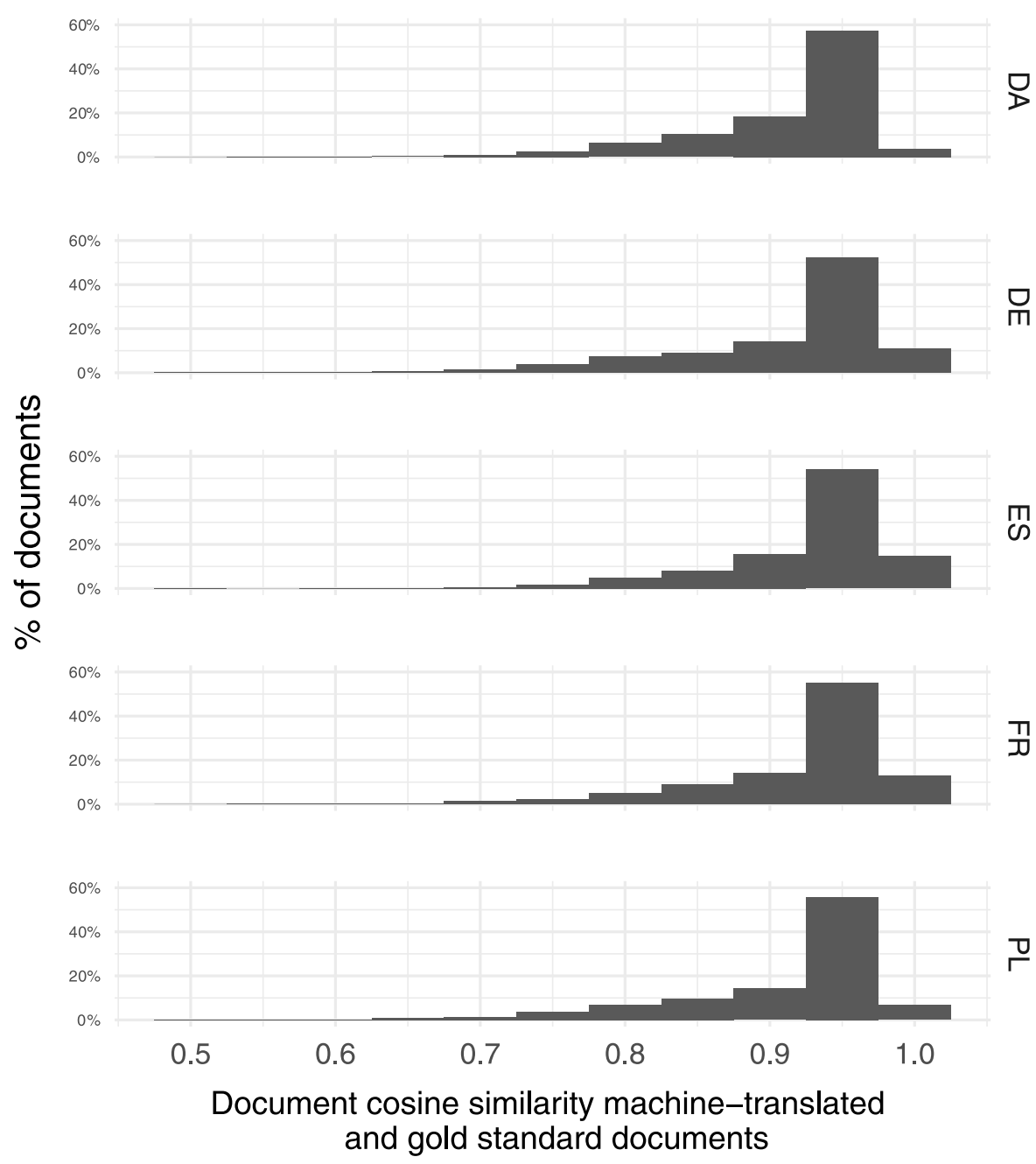

Figure 3. Distribution of cosine similarity per language pair.

The exception is French, and to a lesser extent Spanish. In the Spanish case, more unique features are present in the machine-translated than in the gold standard texts, which indicates that Google Translate adds new features to the texts (by using different English translations for the same Spanish word). Similarly, French translations are simplified (different French words are translated as the same English word). ${ }^{17}$ However, regardless of these differences, both the substantial overlap among features and the high cosine similarity scores for both Spanish and French show that their machine-translated and gold standard TDMs are highly similar.

\section{Comparing topic models}

Each document in our corpus is about one or more topics. Do the topic models with the machine-translated text as input assign the same topics to a document as the topic models with the gold standard translated texts? Figure 5 displays for each language how similar topical prevalence is for each pair of gold standard and machine-translated documents (based on an equal number of topics; for the comparison between unequal number of topics, see the Appendix). These correlations denote the extent to which topical prevalence in individual gold standard and machine-translated documents overlaps. The higher the correlation the more

17 It would be very interesting to see if these unique features are actually caused by inaccurate-but in meaning similartranslations. However, due to the automated nature of all the analyses conducted here, this is not within the scope of the current paper. 

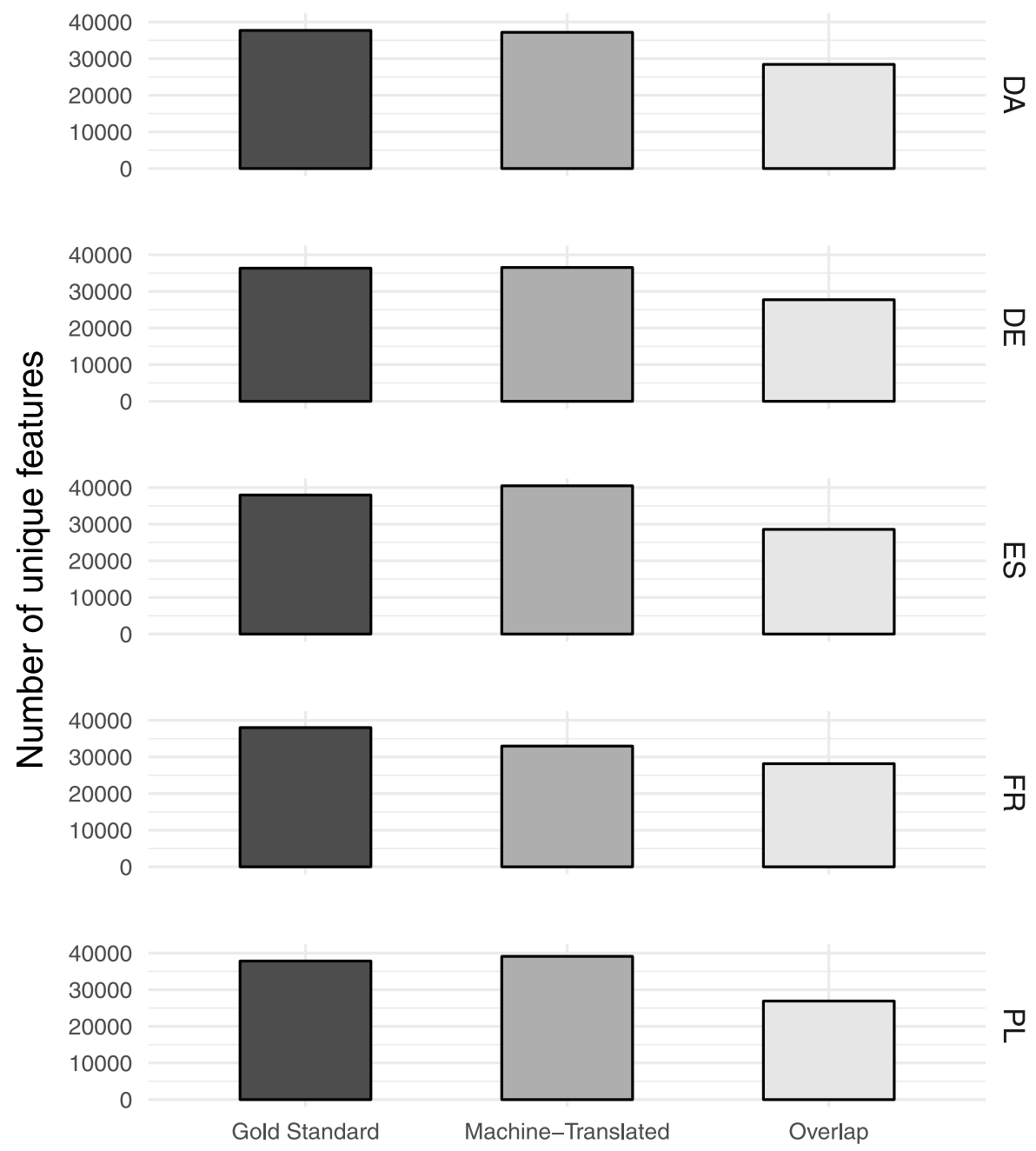

Figure 4. Unique TDM features for gold standard and machine-translated corpora. Reading example: for the French language, the amount of overlapping features is around 28,000, while the total number of features is around 33,000 for the machine-translated documents and around 38,000 for the gold standard documents.

the overlap. ${ }^{18}$ It shows that document-level topical prevalence is similar for gold standard and machine-translated corpora, with on average-across all languages-65\% of document pairs having a topic distribution correlation of 0.8 or higher. Put differently, a particular document is likely to be assigned to identical topics regardless of whether it was machine-translated or gold standard translated.

That said, there are statistically significant differences between languages (see Table 3). ${ }^{19}$ Table 3 breaks down mean topical prevalence for each language, as well as their standard deviations. The highest mean topic distribution per document pair is obtained for Spanish (0.83), and the lowest for French (0.75). Again, the absolute differences are small, and across languages it appears that topical prevalence at the level of individual documents is similar.

Each topic in our data is discussed in several documents. Are these the same documents in the topic models of the machine-translated text and the gold standard translations? To evaluate this

18 Aside from a very small number of documents with negative correlation in case of an unequal number of topics, the overall distributions of correlations in the comparison of unequal models and equal models are similar. This indicates that changing the number of topics toward the optimum for both datasets does not affect document topic distribution scores much.

19 Note that $t$-values for all languages are significant regardless of comparing equal or unequal numbers of topics, so the statistical significance of differences cannot be attributed to a specific language. 


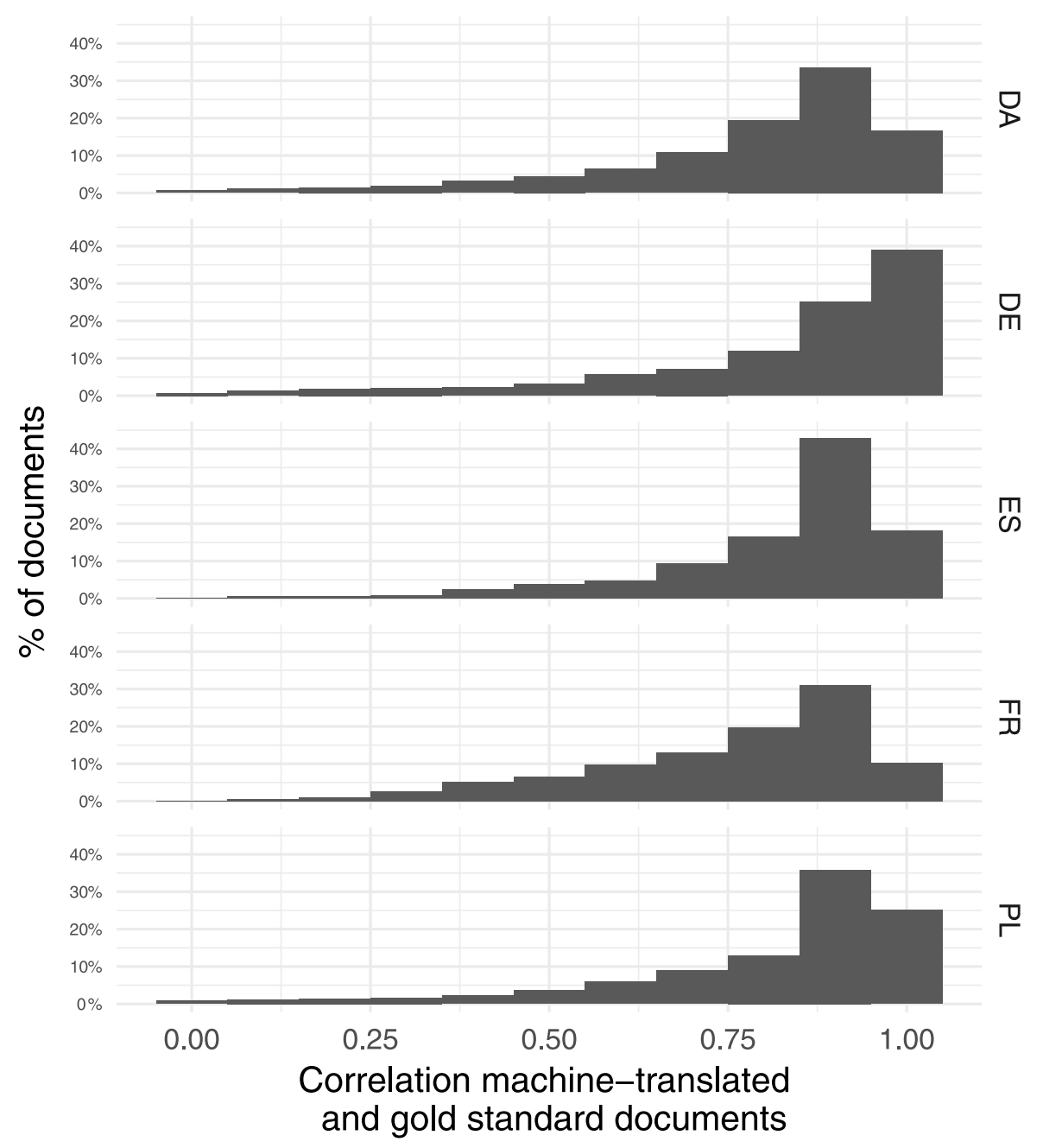

Figure 5. Similarity of document-level topical prevalence with equal number of topics.

Table 3. Similarity of document-level topical prevalence with equal number of topics.

\begin{tabular}{lcccrc} 
Language & N & Mean & St. Dev. & \multicolumn{1}{c}{ Min } & Max \\
Danish & 2,301 & 0.783 & 0.202 & -0.031 & 0.998 \\
German & 2,148 & 0.824 & 0.216 & -0.031 & 0.999 \\
Spanish & 2,335 & 0.826 & 0.165 & 0.028 & 0.997 \\
French & 2,347 & 0.753 & 0.194 & -0.043 & 0.996 \\
Polish & 2,338 & 0.809 & 0.206 & -0.031 & 0.998 \\
Total & 11469 & 0.799 & 0.197 & -0.043 & 0.999
\end{tabular}

Note: ANOVA results: $\mathrm{F}(4,11464)=56.414, \rho<0.001, \eta^{2}=0.019$

we calculate the correlations between the topical prevalence of each topic in the gold standard and the machine-translated documents (Figure 6 show the results of 446 topic distribution comparisons). ${ }^{20}$ As in the case of document-level topic distributions, these corpus-level correlations are generally quite similar, having a mean of 0.69 . This indicates that on average topics are similarly distributed across all documents in the corpus. This indicates that a topic is

2090 comparisons for German, and 89 comparisons for Danish, Spanish, French and Polish, summing to 446. 


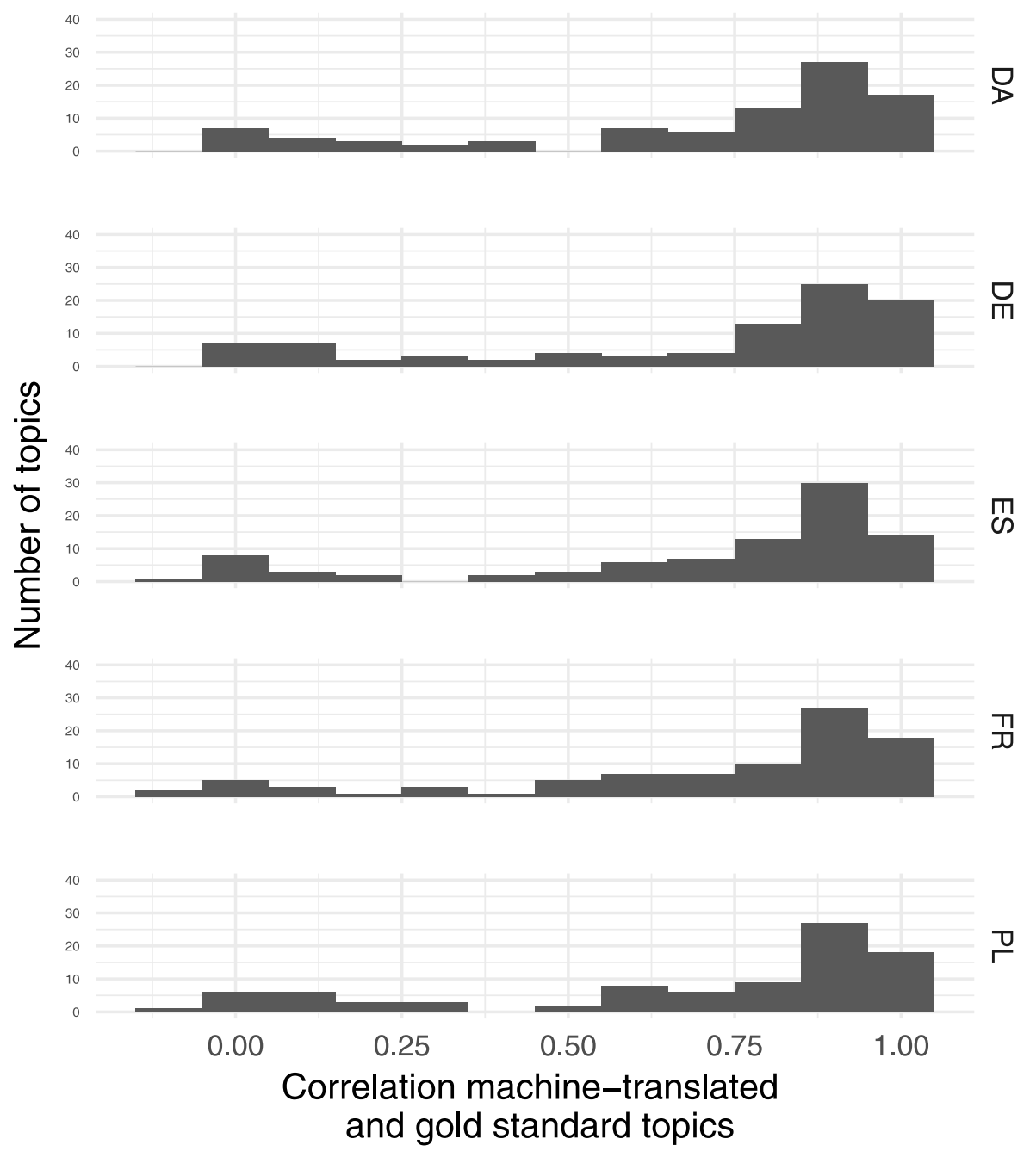

Figure 6. Similarity of corpus-level topical prevalence with equal number of topics. Overall descriptives: $N=446, M=0.699, S D=0.321$.

likely to be distributed similarly across documents, regardless of whether these documents where machine translations or gold standard translations of the same source.

Finally, we also compare the similarity in the content of paired topics. To do so, we analyze for each topic pair the stem loadings of all shared features in the gold standard and machinetranslated TDMs. The results are presented in Figure 7. Again, the average correlation is about 0.70 across languages indicating that topical content, as measured by the distribution of stem loadings, is similar for both the machine-translated and the gold standard corpora. ${ }^{21}$ That implies that topics are discussed using the same terms in both the machine-translation and gold standard translation documents.

\section{Conclusion}

The results in this paper support the claim that Google Translate is a useful tool for researchers using bag-of-words text models for comparative questions. We first found TDMs for machine translations and gold standard translations to be highly similar, with substantively small differences across languages. What is more, we found considerable overlap in the set of features

21 A question that remains is the spike in topic correlations on the low end of Figures 6 and 7 . The reason is that these are topic pairs that contain very few documents. As such these differences are unlikely to affect the topic model output of theoretical interest. Results discussed in the Supplementary Appendix present evidence for this explanation. 


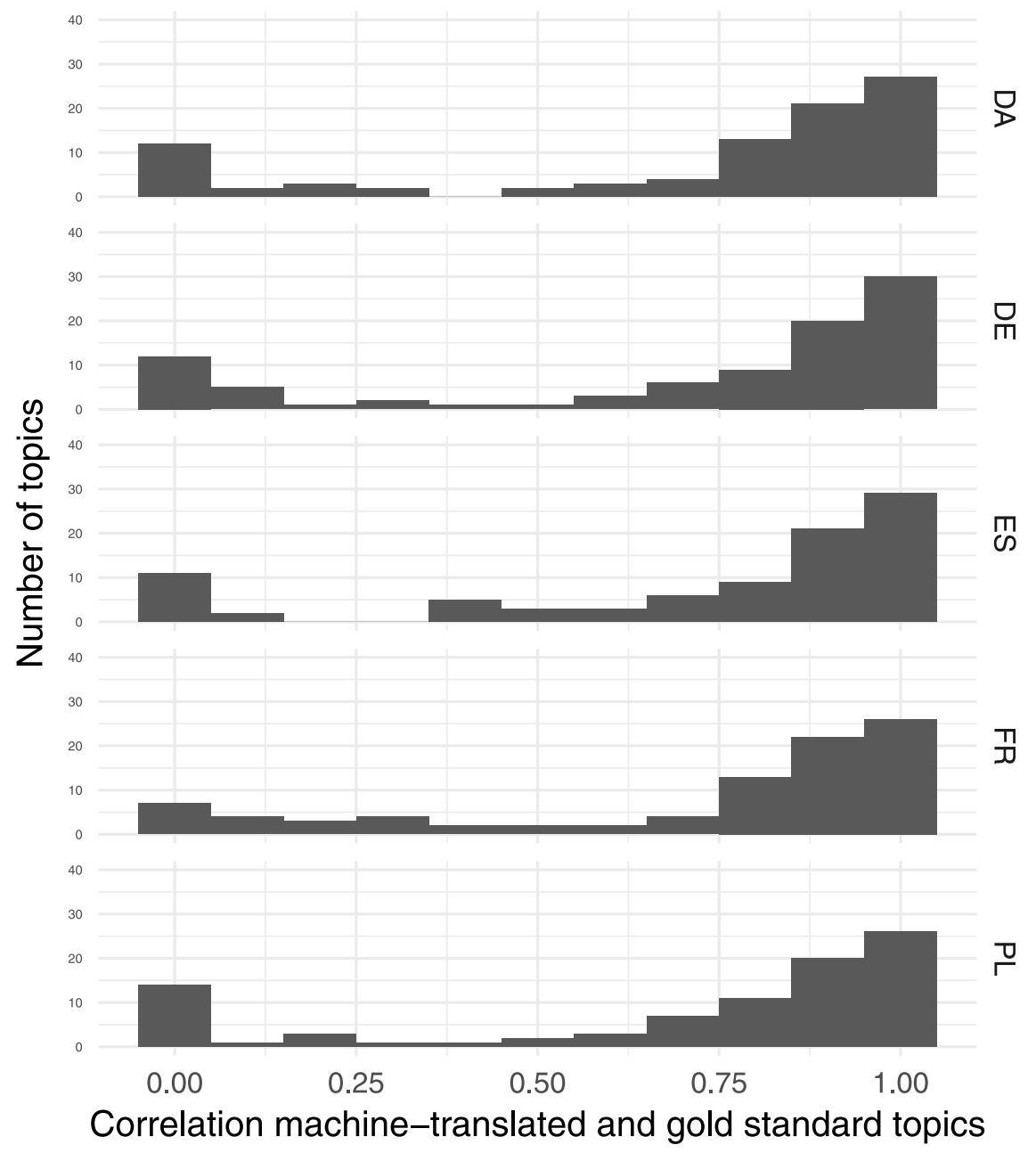

Figure 7. Similarity of topical content with equal number of topics. Overall descriptives: $N=446, M=0.708$, $S D=0.345$.

(stems) generated from both corpora. With regards to LDA topic models, at both the document and the corpus levels we found topical prevalence to be generally similar with only small differences across languages. Furthermore, we found topical content to be highly similar.

Do our findings extend to other bag-of-words approaches such as position scaling or sentiment analysis? If a topic model with 90 models using machine-translated documents is highly similar to the topic model with the gold standard documents, we believe it to be very likely that a 2-dimensional or 3-dimensional scaling model can be similarly reproduced. In addition, for sentiment analysis machine-translation is already used. Sentiment dictionaries are sometimes translated from English to other languages without validation. This is problematic since the specific meaning of words is more relevant. Some words may be translated in such a way that they lack emotional content, while other words may gain emotional content in translation. As long as these translation issues are random, the problem of the identification of false positives or false negatives is reduced when sentiment scores are aggregated over entire documents. Then again, we do not quite know whether these translation issues are random or not. We leave these issues for future work.

\section{Supplementary material}

For supplementary material accompanying this paper, please visit https://doi.org/10.1017/pan.2018.26. 


\section{References}

Agarwal, Apoorv, Boyi Xie, Ilia Vovsha, Owen Rambow, and Rebecca Passonneau. 2011. Sentiment analysis of twitter data. In Proceedings of the workshop on languages in social media. Association for Computational Linguistics, pp. 30-38.

Aharoni, Roee. 2015. Automatic detection of machine translated text and translation quality estimation. PhD thesis, Department of Computer Science, Bar-Ilan University Ramat Gan, Israel.

Balahur, Alexandra, and Marco Turchi. 2014. Comparative experiments using supervised learning and machine translation for multilingual sentiment analysis. Computer Speech and Language 28(1):56-75.

Benoit, Kenneth, and Paul Nulty. 2013. Quanteda: quantitative analysis of textual data. http://quanteda.io. An R library for Managing and Analyzing Text.

Bird, S., E. Klein, and E. Loper. 2009. Natural language processing with Python. O'Reilly Media, Inc.

Blei, David M., Andrew Y. Ng, and Michael I. Jordan. 2003. Latent dirichlet allocation. Journal of Machine Learning Research 3(1):993-1022.

Chuang, Jason, Sonal Gupta, Christopher Manning, and Jeffrey Heer. 2013. Topic model diagnostics: assessing domain relevance via topical alignment. In Proceedings of the 30th international conference on machine learning (ICML-13), pp. 612-620.

Courtney, Michael, Michael Breen, Iain McMenamin, and Gemma McNulty. 2017. Deductive validation of machine translation for text analysis in comparative politics. Working Paper, Dublin City University.

De Vries, Erik, Martijn Schoonvelde, and Gijs Schumacher. 2018. Replication data for: no longer lost in translation: evidence that Google Translate works for comparative bag-of-words text applications, https://doi.org/10.7910/DVN/VKMY6N, Harvard Dataverse, V1.

Denny, Matthew James, and Arthur Spirling. 2018. Text preprocessing for unsupervised learning: Why it matters, when it misleads, and what to do about it. Political Analysis 26(2):168-189.

Gray, Russell D., and Quentin D. Atkinson. 2003. Language-tree divergence times support the Anatolian theory of Indo-European origin. Nature 426:435-439.

Greene, Zac, Andrea Ceron, Gijs Schumacher, and Zoltán Fazekas. 2016. The nuts and bolts of automated text analysis. Comparing different document pre-processing techniques in four countries. Open Science Framework.

Grimmer, Justin, and Brandon M. Stewart. 2013. Text as data: the promise and pitfalls of automatic content analysis methods for political texts. Political Analysis 21(3):267-297.

Hampshire, Stephen, and Carmen Porta Salvia. 2010. Translation and the internet: evaluating the quality of free online machine translators. Quaderns: revista de traducció 17:197-209.

Hornik, Kurt, and Bettina Grün. 2011. Topicmodels: an R package for fitting topic models. Journal of Statistical Software 40(13):1-30.

Kaljahi, Zadeh, and Rasoul Samad. 2015. The role of syntax and semantics in machine translation and quality estimation of machine-translated user-generated content. PhD thesis, Dublin City University. Available at http://doras.dcu.ie/20499/.

Koehn, Philipp. 2005. Europarl: a parallel corpus for statistical machine translation. In MT summit, vol. 5, pp. 79-86.

Koehn, Philipp, and Christof Monz. 2006. Manual and automatic evaluation of machine translation between European languages. In Proceedings of the workshop on statistical machine translation. Association for Computational Linguistics, pp. 102-121.

Loaiciga, Sharid, Thomas Meyer, and Andrei Popescu-Belis. 2014. English-French verb phrase alignment in europarl for tense translation modeling. In LREC, pp. 674-681.

Lotz, Susan, and Alta Van Rensburg. 2014. Translation technology explored: has a three-year maturation period done Google Translate any good? Stellenbosch Papers in Linguistics Plus 43:235-259.

Lucas, Christopher, Richard A. Nielsen, Margaret E. Roberts, Brandon M. Stewart, Alex Storer, and Dustin Tingley. 2015. Computer-assisted text analysis for comparative politics. Political Analysis 23(2):254-277.

Popescu-Belis, Andrei, Thomas Meyer, Jeevanthi Liyanapathirana, Bruno Cartoni, and Sandrine Zufferey. 2012. Discourse-level annotation over europarl for machine translation: connectives and pronouns. In Proceedings of the eighth international conference on language resources and evaluation (LREC). Number EPFL-CONF-192582.

Roberts, Margaret E., Brandon M. Stewart, and Edoardo M. Airoldi. 2016. A model of text for experimentation in the social sciences. Journal of the American Statistical Association 111(515):988-1003.

Scarton, Carolina, and Lucia Specia. 2014. Document-level translation quality estimation: exploring discourse and pseudo-references. In The 17th annual conference of the European association for machine translation, pp. 101-108.

Schwarz, Daniel, Denise Traber, and Kenneth Benoit. 2017. Estimating intra-party preferences: comparing speeches to votes. Political Science Research and Methods 5(2):379-396. 